Recherches en didactique des langues et des cultures

Les cahiers de l'Acedle

11-2| 2014

Plurilinguisme(s) et entreprise : enjeux didactiques

\title{
Adapter les formations en langues aux pratiques langagières plurilingues en milieu professionnel
}

Eve Lejot

\section{(2) OpenEdition}

Journals

Édition électronique

URL : http://journals.openedition.org/rdlc/1759

DOI : $10.4000 /$ rdlc. 1759

ISSN : $1958-5772$

Éditeur

ACEDLE

Référence électronique

Eve Lejot, «Adapter les formations en langues aux pratiques langagières plurilingues en milieu professionnel », Recherches en didactique des langues et des cultures [En ligne], 11-2 | 2014, mis en ligne le 07 juin 2014, consulté le 02 mai 2019. URL : http://journals.openedition.org/rdlc/1759 ; DOI : 10.4000/rdlc. 1759

Ce document a été généré automatiquement le 2 mai 2019.

\section{$\theta \Theta \Theta \Theta$}

Recherches en didactique des langues et des cultures is licensed under a Creative Commons AttributionNonCommercial-NoDerivatives 4.0 International License 


\section{Adapter les formations en langues aux pratiques langagières plurilingues en milieu professionnel}

Eve Lejot

\section{Contexte de recherche}

1 Le plurilinguisme concerne la capacité intrinsèque d'une personne à maîtriser plusieurs langues à des niveaux qui ne sont pas nécessairement identiques, alors que le multilinguisme :

renvoie à la présence dans une zone géographique déterminée - qu'elle soit limitée ou étendue - de plus d'une " variété de langues » (c'est-à-dire le mode d'expression d'un groupe social) reconnue officiellement ou non comme langue; dans une telle zone géographique, certains individus peuvent être monolingues et ne parler que leur propre variété de langue (Beacco \& Byram, 2007, p. 8)

2 Les travaux que nous évoquons dans cet article ont été réalisés dans une organisation et une entreprise ${ }^{1}$ à Hambourg en Allemagne. Ils relèvent de deux aspects : le plurilinguisme des salariés ainsi que le statut accordé aux langues de ces deux environnements professionnels multilingues. Les politiques linguistiques constituent une base nécessaire pour équilibrer le fonctionnement de tout système institutionnel international ; les règles de communication sont instaurées par souci d'efficacité et d'équité linguistique afin d'optimiser la compréhension des documents circulant entre les filiales d'un pays à l'autre; la formation des acteurs à des langues de travail communes permet le maintien d'une cohérence entre les différents sites. Certaines langues ont leur place, d'autres la prennent et d'autres encore la monopolisent. 


\section{Le multilinguisme en milieu professionnel}

3 Les résultats des projets européens (ELAN 2006, LEAP 2009, CELAN 2011, PIMLICO 2011, Dylan 2012), sur les pratiques langagières des entreprises, permettent de partir de faits attestés et de créer les entrées d'analyse permettant d'appréhender plus efficacement nos terrains de recherche. Les études quantitatives ELAN et PIMLICO confirment l'idée selon laquelle dans le domaine du commerce international, l'anglais reste pour beaucoup la langue unique, néanmoins des stratégies de gestion de langues (SGL selon ELAN 2006) peuvent répondre aux exigences du marché du travail européen. Le projet DYLAN apporte une dimension linguistique avec des analyses qualitatives et pluri-méthodologiques; il identifie un décalage entre les politiques linguistiques et les pratiques langagières.

4 Le constat du décalage entre une réalité multilingue et une politique linguistique centrée sur la lingua franca de nombreux milieux professionnels internationaux hambourgeois a été l'élément déclencheur de cette recherche ${ }^{2}$. Deux milieux professionnels où se pratiquent quatre des langues parmi les plus parlées en Europe sont au centre de ce travail de recherche: ce sont les sites respectifs d'une entreprise aéronautique européenne et de l'UNESCO à Hambourg. Ces deux institutions ont cinq points communs : l'utilisation de l'anglais comme langue véhiculaire, la localisation d'un site à Hambourg en Allemagne, leurs sièges sociaux respectifs en France, des communications régulières avec des sites de pays hispanophones ainsi qu'une offre de cours d'espagnol, de français, d'anglais et d'allemand auprès de leurs employés. Grâce à notre position de professeur de français langue étrangère depuis 2007 dans le milieu professionnel et dans des universités privées et publiques, nous sommes au cœur de la variété de l'offre de cours en français, allemand, espagnol et anglais sur Hambourg. Les enseignements en langues ne sont pas abordés complémentairement et on continue à enseigner comme si les apprenants avaient besoin de quatre compétences linguistiques dans une langue cible unique. Nous sommes donc témoin et actrice d'un enseignement qui n'intègre pas les pratiques plurilingues de nos terrains de recherche. Les méthodes appliquées en entreprise paraissent anachroniques à l'heure de la promotion du plurilinguisme en Europe, alors pourquoi ne pas chercher à être complémentaires dans les enseignements plutôt qu'additionnels?

\section{Le multilinguisme en contexte franco-allemand}

On constate une hétérogénéité dans les données susceptibles de réguler les pratiques langagières entre la France et l'Allemagne. En France, la langue française est historiquement protégée comme un monument. En Allemagne le débat sur le statut de l'allemand est ouvert.

En raison de l'internationalité du milieu et de l'influence du concurrent américain de la multinationale située à Hambourg, la suprématie de l'anglais sur le milieu aéronautique est rarement remise en question, alors qu'au sein de l'UNESCO le bilinguisme anglaisfrançais est officiel. Ces deux langues y ont le statut de langues de travail, et à celles-ci s'ajoutent six autres langues officielles également répertoriées. Ces dernières sont pourtant trop peu utilisées lors des événements internationaux.

7 Les faits multilingues sont donc là, mais les politiques linguistiques des entreprises sont souvent méconnues ou non mises en œuvre. Le plurilinguisme est un élément de 
reconnaissance des publics des entreprises et un facteur de performance commercial. Entre les lignes de l'étude ELAN et d'après nos observations à Hambourg en milieu professionnel, on peut saisir le multilinguisme sous deux formes : normée et spontanée. Cet article s'inscrit dans le domaine de la didactique. Il est issu de notre expérience d'enseignement du français en milieu professionnel et fait de même suite à une analyse linguistique et sociologique des discours en milieu professionnel (Lejot 2014). L'objectif de ce travail interdisciplinaire est ici de proposer des alternatives didactiques.

\section{La réalité à Hambourg : travail prescrit/travail effectif}

8 Un bilan des pratiques langagières quotidiennes des deux organisations étudiées va maintenant nous permettre de dessiner l'étroit lien de ce travail entre le champ disciplinaire de l'analyse de discours et celui de la didactique.

\section{A l'Unesco : un plurilinguisme pragmatique}

Le fait que l'ancien directeur ${ }^{3}$ de l'UIL ${ }^{4}$ soit malien francophone, docteur en linguistique n'est pas sans relation avec l'accent qui a été mis sur l'apprentissage des langues entre 2000 et 2009. Ce dernier est un acteur engagé pour la politique du plurilinguisme aussi bien en ce qui concerne les formations en Afrique qu'en ce qui concerne les réunions ou conférences au sein de l'organisation. Il considère que cette "/distribution fonctionnelle » (Entretien directeur UNESCO, L. 51-52) 5 trouve régulièrement ses limites et que les alternances de langues sont courantes dans les locaux de l'UIL ${ }^{6}$ :

quand on doit s'exprimer, quand c'est une écoute, bon donc on comprend, on écoute ; quand on doit exprimer, on est dans une phase active, bon dans ce cas on va formuler soi-même alors là on va dans une langue qu'on maitrise mieux (Entretien directeur UNESCO, L. 76-87)

10 L'accord légal de l'UNESCO évoque un bilinguisme de fait qui ne reflète pourtant pas toujours la réalité, comme l'illustre la marque du pluriel suivante suivie d'une unique langue "Working languageS: English». Cette marque du pluriel, oubliée sur le programme d'une conférence de l'UIL à Hambourg, fait état d'une ambiguïté concernant l'usage de l'anglais omniprésent à l'instar du français également langue de travail. Le directeur a souhaité commenter cet événement lors de notre entretien. Il a expliqué que pour des raisons de restrictions budgétaires, il a dû renoncer à un service de traduction simultanée. L'anglais s'est par conséquent imposé comme langue de communication pour cet événement.

\section{Dans l'entreprise aéronautique : un plurilinguisme inassumé}

11 L'entreprise aéronautique communique difficilement sur sa politique linguistique. Ses stratégies de communication multilingue ${ }^{7}$ sont variées avec par exemple d'un côté une version exclusivement en anglais du site internet et d'un autre côté des newsletters internes diffusées en quatre langues.

L'anglais est un argument de recrutement comme l'explique madame Rachel Schroeder la directrice marketing emploi interrogée sur les opportunités de l'entreprise en $2010^{8}$ :

$\mathrm{Au}$-delà des diplômes, nous recherchons des candidats maîtrisant l'anglais, langue officielle d[e Multinationale]. 
13 En l'occurrence, il existe un document au sein de la multinationale, le AP2080 relatant la nécessité d'une langue commune pour les ingénieurs. Dans ce texte de politique linguistique interne, la partie orale du travail n'est pas abordée mais semble s'inscrire dans cette continuité. Le document nommé "/Reference Language » traite des terminologies à utiliser pour le vocabulaire technique, et explicite les besoins d'un vocabulaire de spécialité commun pour éviter les ambiguïtés :

The purpose of this document is to prescribe the rules to be applied as regards Language and provide a Reference Language Baseline covering terms, expressions and abbreviations that are unambiguous, accurate, widely known, accepted and used. ${ }^{9}$ (Airbus Reference Language AP2080, 2003, p. 1)

14 En raison de la multitude des sites internationaux du constructeur aéronautique, la cohérence et l'efficacité des pratiques langagières sont, à plusieurs reprises, décrites comme primordiales :

Coherent and concise communication in an international company such as [Multinationale] requires that a common language is used. Formal control of a common language is vital for consistency, contractual issues and simply for efficiency. ${ }^{10}$ (Airbus Reference Language AP2080, 2003, p. 1)

15 Ce document est pourtant souvent méconnu des employés. Le langage utilisé est l'anglais et pour l'uniformité du vocabulaire, l'anglais américain est explicitement la langue technique officielle :

The [Multinationale]s language is English using the aeronautical terminology in common use, i.e. American aeronautical terminology. ${ }^{11}$ (Airbus Reference Language AP2080, 2003, p. 4)

Cette précision vaut particulièrement pour des «signifiants » différents pour des «/ signifiés/ » similaires comme " petrol» en anglais britannique pour «/gaz(oline) » en anglais américain. Cette uniformisation concerne également l'orthographe des mots, comme « analyse » en anglais britannique et « analyze » en anglais américain.

17 Il est à noter, qu'en 1998, l'entreprise aéronautique a fait la commande d'un document plus spécifique au domaine pour unifier la terminologie, la syntaxe et les acronymes pour les programmes informatiques sur un projet. Le titre en est «/Controlled language». L'anglais comme langue de communication demande des ajustements.

\section{Des stratégies multilingues entre paradoxes et ambiguïtés}

18 En résumé, les milieux professionnels qui nous occupent comptent chacun des salariés d'origines diverses avec un site en Allemagne à Hambourg et une présence internationale forte. De profils différents, puisque l'un appartient à l'industrie et l'autre est opérateur institutionnel, ils se heurtent cependant tous deux à la gestion de la diversité des langues au travail avec des décalages entre les politiques affichées et la réalité des pratiques.

L'entreprise aéronautique se présente en effet comme une institution monolingue mais traduit certains documents, tandis que l'UIL ${ }^{12}$ a tendance au bilinguisme tout en prônant le multilinguisme sur ces projets externes.

Ces deux institutions représentent des dynamiques linguistiques opposées puisque l'une prône le monolinguisme et se voit contrainte d'offrir ponctuellement en interne des services multilingues, alors que l'autre base une partie de sa communication sur le multilinguisme et essaie régulièrement de contourner la règle pour se concentrer sur des activités monolingues. Cela étant, les contradictions ne sont pas des critiques de notre 
part, il ne semble pas qu'il y ait de stratégie linguistique idéale car la réalité ne se laisse pas définir en une règle fixe. En terme de stratégie financière institutionnelle, il semble plus facile de prôner une langue unique et d'offrir en parallèle des services multilingues au cas par cas, que de suivre le chemin inverse, c'est-à-dire de mener une politique linguistique multilingue, comme c'est le cas de l'UNESCO, et d'organiser ponctuellement des événements monolingues qui sont perçus comme une transgression à la règle par les acteurs de l'organisation.

En repartant des mesures listées par le projet PIMLICO (2011, p. 2), nous allons regarder plus avant quelles solutions sont proposées sur nos deux terrains.

Tableau 1 : Synthèse des stratégies de gestion des langues ${ }^{13}$ à I'UIL et dans la multinationale

\begin{tabular}{|c|c|c|}
\hline Stratégies de gestion de langues & UIL & $\begin{array}{l}\text { Entreprise } \\
\text { aéronautique }\end{array}$ \\
\hline Emploi d'agents locaux pour résoudre les problèmes de langue & Oui & Oui \\
\hline $\begin{array}{l}\text { Création de sites internet comportant des adaptations culturelles } \\
\text { et/ou linguistiques spéciales }\end{array}$ & Oui & Non \\
\hline Recours à des audits linguistiques & $?^{14}$ & ? \\
\hline Recours à des traducteurs/interprètes professionnels & Oui & Oui \\
\hline $\begin{array}{l}\text { Traduction de documentation promotionnelle, commerciale et/ou } \\
\text { technique }\end{array}$ & Oui & Oui \\
\hline $\begin{array}{l}\text { Programmes de formation linguistique et de sensibilisation } \\
\text { culturelle }\end{array}$ & Oui & Oui \\
\hline Apprentissage des langues en ligne & Non & Non \\
\hline Sélection des salariés et politique d'embauche & Oui & Oui \\
\hline Mobilité du personnel & Oui & Oui \\
\hline $\begin{array}{l}\text { Programmes de jumelage avec des collègues étrangers et de } \\
\text { détachement à l'étranger }\end{array}$ & Oui & Oui \\
\hline Création de liens avec les universités locales & Oui & Oui \\
\hline Accueil d'étudiants étrangers en stages & Oui & Oui \\
\hline Embauche de locuteurs de langue maternelle & Oui & Oui \\
\hline $\begin{array}{l}\text { Commerce (diffusion de service) en ligne ayant recours à des } \\
\text { opérations multilingues }\end{array}$ & Oui & Oui \\
\hline $\begin{array}{l}\text { Adaptation du produit ou de son conditionnement aux préférences } \\
\text { et aux coutumes locales }\end{array}$ & Oui & Non \\
\hline
\end{tabular}


Nous voyons dans ce tableau que l'UIL dispose d'un site web multilingue et que la multinationale a un site uniquement en anglais. Une étude des sites web (Guidère, 2008) des compagnies aériennes et des représentations diplomatiques révèle deux tendances distinctes. Les sites à vocation commerciale offrent des versions multilingues alors que les sites des représentations diplomatiques à une échelle nationale sont unilingues, et le contenu de ces derniers oscille entre information et propagande (Guidère, 2008, p. 79). D'une certaine façon, les résultats de cette étude nous montrent que nos terrains de recherche n'appartiennent ni au domaine strictement commercial ni au domaine de la diplomatie au sens de représenter des nations. Ils sont tous les deux face à un public international et là où l'UIL choisit de communiquer sur sa diversité et cherche à atteindre le plus grand nombre, l'entreprise aéronautique s'adresse à une " communauté discursive translangagière $»^{15}$ (Beacco J.-C., 1992, p. 15) unie et ciblée par l'usage de l'anglais ${ }^{16}$.

\section{La compétence plurilingue comme affirmation de la posture professionnelle}

Nous allons maintenant nous intéresser à une stratégie de gestion des langues en particulier : la formation ; et plus particulièrement à l'origine de sa demande.

\section{La nature des attentes}

Pour la mise en place d'une ingénierie de formation, il est nécessaire de définir l'origine de la demande de formation en langue. T. Ardouin distingue quatre motifs de demande :

- la « demande problème ",

- la « demande transfert»,

- la « demande récompense",

- la « demande prétexte » (2003, pp. 51-55).

La « demande problème » vient en réponse à une faille qui reste encore une énigme pour l'entreprise. La formation de langues est ici utilisée comme un moyen détourné pour résoudre un problème de communication ou de relation ou de système ou encore de rendement, dont la source reste inconnue. La «demande transfert" s'inscrit dans le processus traditionnel de la préparation d'un employé qui est dans la situation d'un changement de poste et a besoin de renforcer ou de développer ses connaissances en langue pour ses nouvelles fonctions. La "_demande récompense » intervient suite à une performance professionnelle reconnue pour les employés méritants. Enfin la « demande prétexte " est celle qui sera connotée le plus péjorativement puisqu'elle donnera une excuse à l'entreprise pour entreprendre des restructurations de personnel selon les résultats de chacun à la formation. Il convient donc de se demander si on est face à une demande "/fondée (destinée à résoudre une difficulté ou à adapter sur des postes précis un public migrant) ou [à] une demande de formation "pour la forme" " (Mourlhon-Dallies, 2008, p. 193). La compétence plurilingue (français-anglais ou allemand-anglais ou espagnol-anglais) est souvent une condition de recrutement des secrétaires dans la multinationale. Néanmoins, certaines d'entre elles prennent parfois des cours pour renforcer leur compétence dans la langue d'un nouveau partenaire professionnel. Concernant les ingénieurs, la motivation du cours de langues est souvent liée à une mutation, ce qui explique le pourcentage élevé d'ingénieurs en poste à 
Hambourg apprenant l'allemand pour s'y installer ainsi que $49 \%$ apprenant le français pour préparer une prise de poste à Toulouse. Leur évolution de carrière est favorisée avec la mobilité. Par exemple des programmes internes «trainee » requièrent que les jeunes ingénieurs passent une année dans chacun des quatre pays européens dans lesquels l'entreprise a ses principaux sites (France, Allemagne, Angleterre et Espagne). Enfin les managers de Hambourg apprennent avant tout le français puisque, lorsque leur carrière se développe, ils sont nombreux à faire des allers-retours hebdomadaires au siège social de Toulouse avec un charter quotidien de site à site. Quant à l'apprentissage de l'anglais pour $42 \%$ d'entre eux, il correspond à une volonté de maîtriser au mieux la langue de l'entreprise afin de ne pas perdre la face auprès de leurs collaborateurs.

En bref, les organisations favorisent les stratégies qui leur assurent une image positive et/ ou la prospérité financière sur la scène internationale. Au plan individuel, les salariés sont en quête de reconnaissance auprès de leurs partenaires professionnels et notamment leurs supérieurs comme le montrent les schémas actantiels créés à partir de nos analyses d'entretiens (Lejot, 2014).

\section{L'analyse de discours au service de l'identification des besoins}

Le choix d'utiliser telle ou telle langue dépend étroitement du contexte et de sa perception (Lejot 2014). La question des cadrages (Charolles, 1997) est capitale pour nos travaux puisqu'ils font émerger des tissages de plans ${ }^{17}$ très divers dans l'établissement de la posture professionnelle. Les différents contextes de travail doivent être déclinés dans l'offre de formation car les salariés sont en position mouvante. Arrivée au terme des analyses, nous avons réalisé qu'il n'était pas possible aux salariés de "démêler " les cadres pour extraire des besoins concrets. Il se joue trop de choses au niveau postural sur divers plans professionnels. Dans une moindre part, l'analyse de besoins des 576 employés de l'entreprise aéronautique (Lejot 2014) confirme des contextes d'emploi des langues avec des listes de situations. Les éléments de la sphère personnelle apparaissent comme une motivation très importante de l'apprentissage des langues, les salariés prenant les voyages privés comme justification du besoin de formation. Il est alors difficile pour une école de se positionner entre la sphère personnelle des salariés et les rapports tissés dans le cadre professionnel. Cette situation rend le contenu des formations très complexe à définir.

De plus, dans l'espace de l'activité professionnelle, les variables sont nombreuses (position hiérarchique de l'interlocuteur, objectifs de la discussion, profil linguistique de l'interlocuteur, localisation de l'échange etc.) pour définir une identité professionnelle :

Un même acteur est porteur d'identités professionnelles plurielles (être polyidentitaire), construites par identification à des groupes professionnels d'appartenance et de référence qui se différencient à partir de langage et de code communs, de pouvoir au sein de l'organisation, de valeurs et de fonctions sociales. L'acteur mobilise l'une ou l'autre de ses identités professionnelles selon, d'une part un processus de «zapping identitaire » en fonction de la situation d'interaction où il se trouve ou de la représentation qu'il s'en fait et d'autre part selon un processus d' « assignation identitaire » mobilisé par l'Autre. (Blin, 1997, p. 184)

Nous ne sommes plus des formateurs en langues dans les milieux professionnels, mais nous sommes des formateurs en communication du travail. 


\section{Vers une didactique du plurilinguisme en milieu professionnel} comment ajuster les formations au français, à l'espagnol et à l'allemand qui ont un rôle complémentaire, et essentiel à l'anglais utilisé comme lingua franca sur nos deux terrains de recherche?

Nous allons maintenant brièvement présenter un état des lieux de l'existant didactique sur nos deux terrains de recherche puis nous envisagerons quatre options didactiques pour intervenir sur ces terrains professionnels. La première option est davantage celle d'un professeur de langue puisqu'elle concerne les approches plurielles. Quant aux options qui suivent, elles sont issues de projets émergents. Ainsi, la seconde option serait plutôt celle d'un agent de la communication professionnelle puisqu'elle demande à l'enseignant de didactiser l'environnement de travail. La troisième option ainsi que la quatrième correspondent davantage à la lignée de la recherche-action avec un formateur qui se positionne comme un accompagnateur à la professionnalisation en dépassant le domaine linguistique pour se plonger dans des mécanismes du fonctionnement quotidien des salariés. Enfin nous proposerons une ingénierie de formation envisageable pour nos terrains de recherche.

\section{L'existant en milieu professionnel à Hambourg}

Dans cette section, nous allons tout d'abord traiter brièvement les livres à visée professionnelle, puis le «blended learning » ainsi que la plateforme multilingue Speak up !

\section{Les livres à visée professionnelle}

Pour répondre aux besoins des apprenants, l'enseignement de l'anglais fait l'objet d'une industrie éditoriale abondante et traite aussi bien des pratiques langagières que du concept de communication dans les entreprises. Les réunions et les négociations y sont décryptées et exploitées par tous les médias disponibles en cours. Nos collègues enseignants anglophones en semblent satisfaits. Ils sont les seuls parmi l'équipe pédagogique à employer le terme de "/customers/ $»^{18}$ ou de "clients " pour leurs apprenants, ils évoluent dans une sphère des affaires très demandeuse et se positionnent plus en coach de communication qu'en professeur de langue. Les livres à visée professionnelle utilisés par les professeurs pour chacune des quatre langues proposent des modules exhaustifs et monolingues et ne marquent pas de différences dans l'enseignement de l'anglais majoritaire et celui de l'espagnol, de l'allemand et de l'espagnol.

La contextualisation est en fait une béquille à la qualité de la transversalité. A ce sujet $\mathrm{F}$. Mourlhon-Dallies considère que : le très faible degré de contextualisation des besoins des publics concernés rejette les spécialités en toile de fond, ce qui conduit à une sorte de transversalité "/par défaut", partiellement compensée par un effort de contextualisation dans le travail (2008: 45). C'est un système d'enseignement à la carte. F. 
Mourlhon-Dallies remarque que: "partout la spécialité est volontairement effacée " $(2008,91)$. proposé par le groupe Speak up! qui a développé une plateforme professionnelle multilingue mis en place notamment au sein de la multinationale aéronautique de Hambourg en 2012. La plateforme multilingue Speak up !19 est financée avec le soutien de la Commission européenne et est réalisée par une équipe répartie aux quatre coins de l'Europe. Son objectif est de faciliter la mobilité des jeunes travailleurs. La plateforme propose des formations en six langues : anglais, français, espagnol, portugais, italien et allemand. Les différentes formations sont élaborées à partir d'une langue et sont traduites dans les cinq autres par une équipe de traducteurs professionnels. L'idée est que les participants à une formation pour un métier particulier puissent naviguer d'une langue à l'autre s'ils le souhaitent. La progression à partir du niveau débutant est constituée de dix leçons qui proposent des points de grammaire, de vocabulaire et d'expression utilisés dans le corps de métier ciblé. Chaque cours comprend :

- Dix leçons, y compris un aperçu général,

- Une terminologie spécifique au métier en question,

- Des expressions de la vie quotidienne,

- Des informations pratiques sur l'environnement professionnel. (News letter Speak up !, Avril 2012) 
40 personnel qualifié de notre travail de recherche se passent néanmoins en présentiel et nous avons $\mathrm{vu}$, avec la réaction mitigée sur l'apprentissage en ligne basé sur l'intercompréhension, que cette formule semble difficilement envisageable auprès de notre public. Cependant, nous retenons du projet Speak up! la proposition d'un apprentissage de langues à la carte et d'une spécialisation des apprenants dans le vocabulaire qui leur est le plus utile selon les langues. Même si la plateforme est destinée à développer une langue choisie par l'apprenant pour la préparation d'un échange professionnel dans un pays cible, ce qui nous intéresse dans ce projet, c'est de pouvoir naviguer d'une langue à l'autre à partir d'un même contenu sur son activité de travail. Le concept proposé est multilingue et s'adresse à un public plurilingue. Les formateurs qui ont créé ces plateformes ont passé plusieurs jours en immersion dans les milieux professionnels ciblés. B. Brödermann, responsable pour le développement de la formation en ligne des deux métiers sur le site de la multinationale de Hambourg a donc collecté des données sur place pour l'analyse du travail des apprentis ${ }^{20}$. B. Brödermann est une enseignante en français, en allemand et en anglais, et elle a récemment suivi une formation en médiation. Il semblerait que, dès l'instant où les formateurs de langues en entreprise veulent concevoir des programmes ou faire en sorte d'être au plus proche de la réalité de leurs apprenants, ils cherchent des réponses dans la médiation, la communication ou les processus qualité de l'organisation de travail. Chacun essaie de façonner son profil pour être mieux armé pour aborder les problématiques institutionnelles.

Ces propositions se croisent sur les terrains, mais elles restent encore trop éparses et ne parviennent pas concrètement à s'éloigner de l'apprentissage langue par langue. Nous allons maintenant voir si ce qui a été développé en didactique plurilingue serait adaptable au milieu professionnel.

\section{Les approches plurielles}

La première option est traditionnelle puisqu'elle est basée sur un double travail de sensibilisation linguistique et culturelle. Les approches plurielles proposent des modes d'enseignement intégrant plusieurs langues et plusieurs cultures. Ces approches sont pensées par des didacticiens des langues et diffusées en milieu scolaire ainsi que lors de formations des enseignants. Elles n'émanent en rien des entreprises. En effet, l'âge de la didactique monolingue laisse place petit à petit à la didactique du plurilinguisme et depuis quelques années aux approches plurielles. M. Candelier situe la première utilisation du terme «/approche plurielle» au singulier pour qualifier «l'éveil aux langues » lors de la publication d'une lettre de l'ACEDLE (2002). Nous allons cependant voir maintenant si elles y seraient applicables. Les approches plurielles sont au nombre de quatre: l'approche interculturelle, l'éveil aux langues ${ }^{21}$, la didactique intégrée des langues et l'intercompréhension. Nous allons écarter l'éveil aux langues car c'est un programme de sensibilisation à la diversité linguistique et culturelle destiné aux enfants. En revanche les salariés de nos deux terrains d'investigation participent à des formations en communication interculturelle. Nous sommes tout à fait conscients que cette facette interculturelle est primordiale, mais ce n'est pas une facette qui devrait continuer à être exploitée isolément. Au contraire elle peut être intégrée à la formation dite linguistique, voire plutôt même communicationnelle ${ }^{22}$. Nous écartons par conséquent ici également 
cette approche interculturelle «isolée » pour nous concentrer sur la combinaison linguistique et professionnalisante.

La didactique intégrée des langues nous intéresse de prime abord par la possibilité qu'elle offre de combiner des langues. Chaque nouvelle langue joue un rôle de pivot pour intégrer la suivante. E. Roulet a développé des travaux sur la didactique intégrée des langues dès le début des années 80 . Cette pratique est en adéquation avec les attentes plurilingues, cependant son dispositif est davantage adapté au contexte scolaire qui assure une continuité dans le passage entre les langues. Les modules de langues distillés irrégulièrement et au compte-gouttes par les institutions professionnelles ne pourraient pas répondre aux exigences de temps d'un tel processus.

Dès nos premiers pas sur nos terrains d'investigation, nous avons pensé que l'approche de l'intercompréhension serait la plus adaptée à des milieux professionnels où quatre langues majeures se côtoient. Cette approche pourrait être une solution pour combler les déficiences linguistiques de manière ciblée dans le contexte professionnel. Relativement reconnue en contexte universitaire et en matière de politique linguistique européenne, l'intercompréhension est encore à l'étude pour les entreprises. Le programme Lifelong Learning du conseil de l'Europe finance les projets Prefic ${ }^{23}, \mathrm{CINCO}^{24}$ et Intermar ${ }^{25}$. Ces derniers ciblent la formation d'apprenants à l'interaction multilingue dans les milieux professionnels internationaux. Ces travaux sont en cours de développement vers une dimension orale interactive qui est encore peu approfondie. Idéalement, la communication basée sur l'intercompréhension réduirait les risques de malentendus puisque la majorité des salariés pourrait s'exprimer dans sa langue maternelle. Ceci nécessiterait une excellente maîtrise de la part de chacun en compréhension orale et écrite de plusieurs langues par les salariés. Cette approche pourrait être envisagé pour travailler sur des compétences partielles et pour créer des modules sur objectifs spécifiques du type : «/comprendre les apartés de vos voisins de réunion en $20 \mathrm{~h}$ !» ou encore «Lisez le par l'écrit ${ }^{26}$ pour que plus aucune information sur post-it ou emails expéditifs ne vous échappe ! ». Les langues cibles pourraient être couplées pour rendre la démarche plus novatrice auprès des décideurs. Ces formules demanderaient à être développées avec du matériel authentique et répondraient à des besoins spécifiques.

\section{Une approche par la médiation}

La deuxième option consiste à transformer l'environnement de travail en organisation apprenante par le biais de la médiation. Nous pensons que la médiation est un bon moyen d'enseigner en dehors de la classe (ou du bureau en cours particulier) en sortant du format classique professeur/élèves. Cette manière de faire permet de confronter les situations d'apprentissage de la langue cible à l'environnement de travail du salarié, de réfléchir sur son profil langagier de manière indirecte ainsi que sur ceux de ses collègues. La médiation permettrait d'aider à créer de nouveaux modes de communication entre collègues pour favoriser l'apprentissage de la langue locale par exemple. Jusqu'ici, les analyses d'entretiens ont néanmoins montré que certains salariés considèrent comme une perte de temps de parler dans leur langue maternelle à des collègues qui cherchent à l'apprendre. De plus, nous avons vu que parler une autre langue que la lingua franca les fait sortir de leur rôle professionnel, ce qui les met mal à l'aise dans leur posture professionnelle. $\mathrm{Au}$ vu des résultats, nous considérons qu'instaurer une approche par la 
médiation poserait des problèmes de posture professionnelle et remettrait en question la norme linguistique de nos deux terrains d'étude.

\section{L'apprentissage par le travail}

La troisième option didactique est de considérer le formateur en langue comme un accompagnateur à la professionnalisation. Le projet « Learning through work» (Newton, Miller, Bates, Page, \& Akroyd, 2006 ; Braddell, 2007) implique que le formateur se rende sur le lieu de travail auprès des employés dans leur quotidien afin de mieux comprendre la place des personnes à qui il enseigne dans leur environnement de travail. Cette pratique nous semble adaptée à des professions de bas niveau de qualification, mais elle fait entrer en jeu trop d'exposition d'un besoin d'apprentissage au regard des collègues dans les bureaux. Les relations de bureau se basent plus sur des compétences abstraites, il n'y a pas de résultats avec un produit final palpable ou un projet quantitativement évaluable comme pour les métiers manuels. Dans ces conditions, il serait difficile d'assumer la présence d'un professeur de langue à leur côté, puisque sa simple présence pointerait une faiblesse vis-à-vis des autres salariés.

\section{L'analyse de la communication organisationnelle}

Enfin dans la quatrième option, le formateur met l'accent sur la langue professionnelle. Il est, dans ce cas, entre le domaine linguistique et le domaine de l'analyse des méthodes de travail. Il peut relever des incidents et des critiques grâce à la récolte de témoignages enregistrés. Cette démarche permet d'englober la tâche en fonction de la posture professionnelle. M. Grünhage-Monetti (2007, pp. 34-35) s'appuie sur les trois dimensions de Green (1997) pour rendre la formation en langue plus opérative sur le lieu du travail. Les trois dimensions sont: la dimension opérationnelle ou fonctionnelle, la dimension culturelle et la dimension critique. Elles sont toutes les trois appliquées lors de réalisations authentiques. L'analyse des réunions a fait émerger trois relations :

- d'aide,

- à l'autorité

- aux aspects pratiques. (Lejot, 2014, p. 338)

La dimension opérationnelle apparaît comme la plus marquante puisqu'elle passe par la partie visible de l'activité de travail, c'est-à-dire la langue : " la maîtrise des éléments de la fonction opérationnelle entre dans la représentation commune de ce que doit comporter un cours de langue» (de Ferrari, 2006). Les salariés plurilingues peuvent donner plus de corps à cette dimension en allant au-devant de leurs collègues. La dimension culturelle n'est pas toujours remarquable dans les réunions puisque ce contexte précis est particulièrement normé et que l'institution forme en elle-même une «/communauté de pratique/» (Wenger, 1998) avec sa propre « culture » et donc avec ses propres normes implicites. La relation hiérarchique est le résultat de normes culturelles auxquelles s'ajoutent celles de l'institution professionnelle. En ce sens, la relation hiérarchique couvre les deux pans cités ci-dessus de la dimension culturelle. Cette dernière correspond également à la capacité à communiquer dans un certain contexte : "/C'est le volet de la pertinence des genres» (Grünhage-Monetti, 2007, p. 35). Cette dimension est naturellement omniprésente dans les milieux professionnels internationaux. Elle implique l'intégration des salariés à la culture d'entreprise afin 
d'évoluer professionnellement. La dimension critique est l'élément en fin de chaîne montrant que si les caractéristiques linguistiques et contextuelles tendent vers une interprétation adéquate et commune, elle «donne aux apprenants les instruments permettant d'évaluer la situation sociale de la langue dans un contexte donné (to make sens), de saisir signifié et signifiant» (Grünhage-Monetti, 2007, p. 36). Son rôle est indispensable pour le bon enchaînement des tâches professionnelles. L'objectif est donc dorénavant que les formateurs passent du temps en amont du cours dans l'entreprise ou l'organisation et qu'ils aient la capacité d'analyser le travail de leurs apprenants individuellement pour pouvoir commencer. C'est dans cette seconde phase que l'analyse de discours a encore beaucoup à apporter.

Pour pouvoir passer des cours traditionnels à l'accompagnement à la professionnalisation, deux points clés sont à améliorer : l'un concerne la formation des formateurs, le second est la prise de conscience des apprenants de leurs besoins (Grünhage-Monetti, 2007, p. 43). Nous allons maintenant proposer une formation en quatre étapes pour les salariés de milieux professionnels internationaux.

\section{Une formation en 4 étapes}

En effet, en plus d'avoir une formation en didactique des langues et en interculturalité, le formateur doit aujourd'hui être préparé à explorer la communication professionnelle et à en analyser les rouages. Les écoles prestataires de langues comptent parmi leurs formateurs des diplômés en commerce, en communication ou en économie qui ne sont pas professeurs de langues et des professeurs de langues qui ne sont pas des experts en communication. Ce déséquilibre vient du fait que les écoles ont identifié un problème sur lequel elles ne posent pas de mots et qu'elles ne savent pas résoudre. Le second levier indispensable est la motivation des apprenants et cela passe, comme le décrit M. Grünhage-Monetti (2007), par la conscientisation de ce besoin. Une grande partie des apprenants de français, d'allemand et d'espagnol place leurs besoins de formation en langue au niveau de la sphère personnelle. Ces employés refusent de pointer une incapacité langagière au travail. Les salariés sont dans le déni de leurs besoins. En comparaison, les étudiants des écoles de commerce sont des publics beaucoup plus souples lors des formations. Ils suivent une formation en alternance et ne se sentent pas encore remis en cause dans leur posture professionnelle. Ils sont en demande. Ils cherchent à multiplier leurs compétences et ils seraient des sujets idéaux pour développer un programme à partir de l'analyse de travail d'une des entreprises où ils sont en apprentissage pour, par la suite, étoffer le concept auprès des salariés de nos terrains d'investigation.

51 Nous conceptualiserions la formation en quatre étapes comme le montre le schéma suivant : 


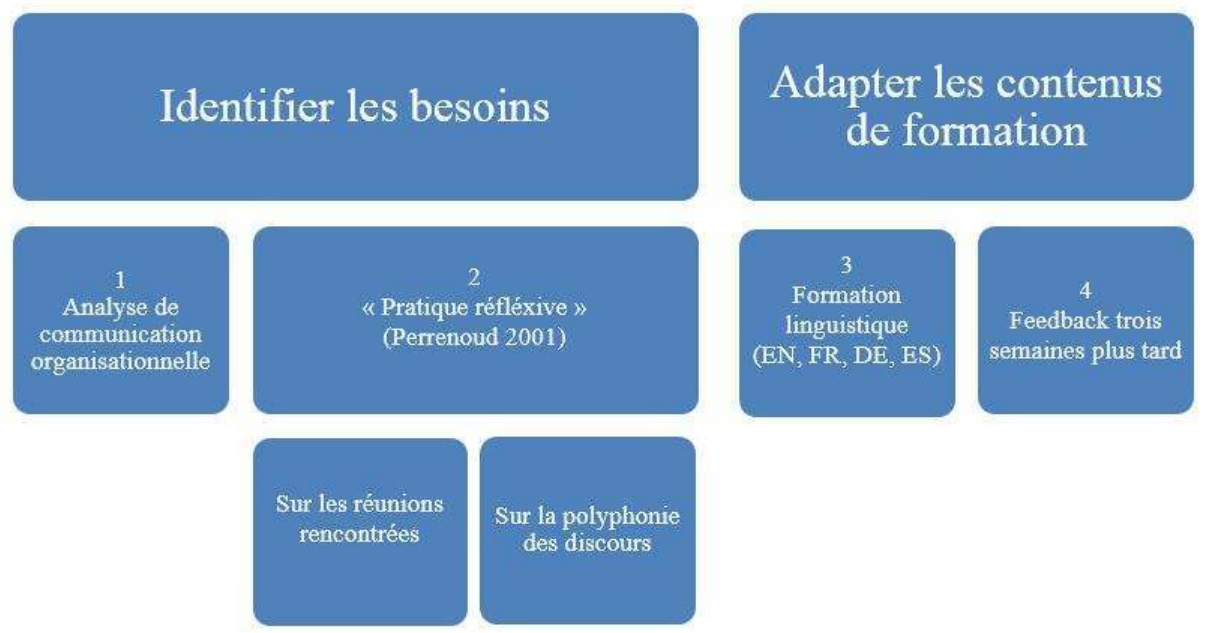

52 Il s'agit tout d'abord d'identifier les besoins. Par conséquent, grâce à l'observation ethnographique et à l'analyse des discours, la première étape consiste en la présence du formateur dans un département donné d'une institution pour procéder à une analyse de communication organisationnelle par poste. La seconde phase réunit les salariés sélectionnés pour la formation pour une phase d'une dizaine d'heures de conscientisation sur leur charge de travail individuel et leurs activités multilingues quotidiennes. Cette étape serait programmée dans la langue commune du groupe. Elle est basée sur l'idée de la «pratique réflexive » (Perrenoud 2001) et pourrait être l'occasion de travailler sur la polyphonie des discours sur la politique linguistique de l'organisation ainsi que sur le rôle des compétences linguistiques dans les réunions. L'objectif de cette étape serait finalement que chaque employé élabore une carte de réseau social avec les profils langagiers de ses interlocuteurs afin de détecter les zones dans lesquelles une sécurité linguistique dans une langue définie favoriserait une qualité communicationnelle. Pour la troisième phase, le groupe est divisé en quatre selon les langues (allemand, anglais, espagnol et français) à acquérir en accord avec les tâches de travail. Une fois cette phase de conscientisation passée, les salariés pourraient participer successivement à différents groupes de langues pour atteindre des "/objectifs spécifiques" liés directement à leur poste et par conséquent à leur carrière. Enfin, une phase de feedback serait organisée à la fin de chaque session pour mesurer les bénéfices directs de la formation et envisager les modifications du quotidien professionnel grâce aux compétences récemment acquises. L'objectif est que finalement les salariés évaluent l'impact de leur sortie de " rôle normé » lorsqu'ils utilisent un autre vecteur de communication que la lingua franca. Chaque salarié sait inconsciemment qu'il se présente différemment selon la langue employée. Il joue un rôle en anglais et sort de son rôle institutionnalisé pour endosser un autre rôle de lien social ou également technique selon le profil linguistique de ses collègues, dans d'autres langues. Les individus adaptent leurs pratiques langagières selon les facteurs temporels, spatiaux et du profil langagier de leur interlocuteur. Ce don d'« ubiquité plurilingue " permet de gérer le flux d'information et donc de maîtriser des différentes perspectives sur une situation de travail. Il permet aussi de gérer les rapports de faces et donc d'établir des relations plus stables avec ses collègues, fournisseurs et clients. Les salariés le souhaitant pourraient poursuivre la procédure d'apprentissage avec un groupe travaillant sur une autre langue cible. Enfin le fait de pratiquer plus de langues des filiales 
de l'organisation facilite la mobilité interne et offre par conséquent de plus nombreuses perspectives de carrière.

\section{Conclusion}

Le déroulement de formation présenté ci-dessus est succinct car nous sommes consciente qu'il ne présente pas de dimension particulièrement novatrice par rapport à l'ACO. Cette option semble coûteuse, mais n'est-ce pas la manière de procéder des consultants qui viennent auditer le fonctionnement d'une entreprise avant de dispenser un séminaire aux employés? Les études ELAN, PIMLICO et DYLAN n'ont-elles pas déjà démontré les bénéfices du plurilinguisme sur les activités commerciales et plus généralement institutionnelles? Si les formateurs peuvent montrer aux entreprises qu'ils sont en mesure de parler leur langue (professionnelle) et donc de dispenser des formations en communication ciblées sur les pratiques langagières en contexte (mais n'est-ce pas la définition d'un cours de communication?), alors les entreprises auront également des fonds pour des analyses au cas par cas et pourront ainsi développer les compétences plurilingues de leurs salariés.

\section{BIBLIOGRAPHIE}

Anscombre, J.-C., \& Ducrot, O. (1983). L'argumentation dans la langue. Bruxelles : Mardaga.

Ardouin, T. (2003). Ingénierie de formation pour l'entreprise. Analyser, concevoir, réaliser, évaluer. Paris : Dunod.

Beacco, J.-C. (1992). " Les genres textuels dans l'analyse du discours : écriture légitime et communautés translangagières". Langages, $\mathrm{n}^{\circ}$ 105, pp. 8-27.

Beacco, J.-C., \& Byram, M. (2007). Guide pour l'élaboration des politiques linguistiques éducatives en Europe. De la diversité linguistique à l'éducation plurilingue. Strasbourg : Conseil de l'Europe.

Blin, J.-F. (1997). Représentations, pratiques et identités professionnelles. Paris: L’Harmattan.

Braddell, A. (2007). Learning through work: developmental on-the-job learning as a vehicle to widen participation in workplace learning. Position paper for the Dept of Health Widening Participation Unit.

Candelier, M. (2002). "Une autre voie pour les langues à l'école primaire : faire de la diversité un instrument d'éducation ". La Lettre de l'Acedle. $n^{\circ}$ 10, article écrit pour Le Monde, non publié, 2001

Charolles, M. (1997). "L'Encadrement du Discours, Univers, Champs, Domaine et Espaces". Cahiers de Recherche linguistique, $\mathrm{n}^{\circ}$ 6, pp. 1-73.

De Ferrari, M. (2006). "Développer la formation linguistique au titre de la formation professionelle continue en entreprise". Migrations études, vol. 133, pp 1-12. 
Degache, C. (1997). "Développer l'intercompréhension dans l'espace linguistique roman : le programme Galatea/Socrates". Assises de l'enseignement du et en français, séminaire de Lyon : Aupelf-Uref.

Ducrot, O. (1980). "Analyse de textes et linguistique de l'énonciation". In O. Ducrot et. al., (dir.). Les mots du discours. Paris: Minuit, pp. 7-56

DYLAN project. (2011). DYLAN project booklet: main findings. Bruxelles : 6ème Programme-cadre de l'Union européenne.

Étude ELAN. (2006). Incidences du manque de compétences linguistiques des entreprises sur l'économie européenne. Bruxelles : Commission Européenne.

Ferrer Mora, H. (2000). "Auf der Suche nach spanischen Modalpartikeln : pero und pues als pragmatische Konnektoren. Stand der kontrastiven Partikelforschung Deutsch-Spanisch". Revista de Filología Alemana, no 8, pp. 253-271.

Green, B. (1997). Literacy information and the learning society. Darwin: Joint conference of the Australian Association and the Australian School Library Association.

Grünhage-Monetti, M. (2007). "Former en langue des salariés immigrés dans l'entreprise : nouvelles orientations didactiques". Le français dans le monde Recherches et applications, $\mathrm{n}^{\circ} 42$, pp. 32-44.

Guidère, M. (2008). La communication multilingue. Traduction commerciale et institutionnelle. Paris et Bruxelles : De Boeck.

Lang, E., \& Umbach, C. (2002). "Kontrast in der Grammatik : spezifische Realisierungen und übergreifender Konnex". In Steube, A. (dir.). Sprachtheoretische Grundlagen der Kognitionswissenschaft : sprachliches und nichtsprachliches Wissen. Leipzig : Linguistische Arbeitsberichte, $\mathrm{n}^{\circ}$ 79, pp. 145-186.

Lejot, E. (2014). Pratiques plurilingues en milieu professionnel international. Frankfort : Peter Lang.

Mourlhon-Dallies, F. (2008). Enseigner une langue à des fins professionnelles. Paris : Didier.

Newton, B., Miller, L., Bates, P., Page, R., \& Akroyd, K. (2006). "Learning through Work: Literacy, language, numeracy \& IT skills development in low-paid, low-skilled workplaces: Literature Review". Institute for Employment Studies, Research Report, $\mathrm{n}^{\circ} 433$.

Perrenoud, P. (2001). Développer la pratique réflexive dans le métier d'enseignant. Professionnalisation et raison pédagogique. Paris : ESF.

Picard, F. (2003). Airbus Reference Language AP2080. Blagnac : Airbus SAS.

PIMLICO, Projet. (2011). Rapport sur les stratégies de gestion des langues et les meilleures pratiques dans les PME européennes : PIMLICO summary. Bruxelles: Commission européenne.

Spaggiari, L., Beaujard, F., \& Cannesson, E. (2005). "A controlled language at Airbus". In Cardey, S., Greenfield, P. \& Vienney, S. (dir.). Machine Translation, Controlled Languages and Specialised Languages: Special issue of Lingvistica Investigationes XXVIII. Amsterdam: John Benjamins, pp. 107-122.

Wenger, E. (1998). Communities of practice: learning, meaning and identity. Cambridge: Cambridge University Press.

Weydt, H. (1983). "Aber, mais und but". In Weydt, H. (dir.). Partikeln und Interaktion. Tübingen : Niemeyer, pp. 148-159. 


\section{NOTES}

1. Que nous désignerons anonymement par le terme « Multinationale ».

2. Des pré-enquêtes ont été menées en 2008 auprès de collègues professeurs de français en entreprise, de salariés de différents secteurs d'activité à Hambourg, de représentants de la chambre de commerce et de la diplomatie française à Hambourg.

3. De 2000 à 2009.

4. UIL est l'acronyme pour UNESCO Institute for Lifelong Learning (Institut de l'apprentissage tout au long de la vie). L'UIL est l'antenne de l'UNESCO à Hambourg.

5. L'entretien avec le directeur de l'UNESCO a eu lieu le 27/10/2010.

6. L'Institut de l'UNESCO pour l'apprentissage tout au long de la vie.

7. La stratégie de communication multilingue est «l'adoption planifiée d'une gamme de techniques visant à permettre une communication efficace avec les clients et les fournisseurs à l'étranger » (Étude ELAN, 2006, p. 6)

8. http://www.studyrama-emploi.com/home_interview.php?id=2012\&handicap=1, dernière visite le 15 janvier 2014

9. Notre traduction: Le but de ce document est de prescrire les règles à appliquer en ce qui concerne la langue, et de fournir une base de référence du langage qui recouvre des termes, expressions et abréviations qui sont sans ambiguïé, précises, largement connues, acceptées et utilisées.

10. Notre traduction: Une communication cohérente et concise dans une compagnie internationale telle que [Multinationale] requiert l'usage d'une langue commune. La maîtrise officielle d'une langue commune est essentielle pour la cohérence, les aspects contractuels et tout simplement pour l'efficacité.

11. Notre traduction: La langue de [Multinationale] est l'anglais, qui emploie la terminologie aéronautique d'usage, à savoir la terminologie aéronautique américaine.

12. Site hambourgeois de l'UNESCO.

13. «Stratégies de communication multilingue " (Étude ELAN, 2006, p. 6) ou «stratégies de gestion des langues » (PIMLICO Project, 2011, p. 1)

14. Compte tenu de la dimension de ces organisations internationales et de la confidentialité liée à certains pans de leur mode de fonctionnement, il nous est difficile de répondre ici par l'affirmative ou par la négative.

15. J.-C. Beacco désigne « une communauté fortement fondée sur une institution (entreprise multinationale, organisme international, domaine scientifique et universitaire, domaine professionnel internationalisé) dans laquelle les statuts des scripteurs sont fixés, la circulation des écrits descriptible, mais qui fonctionnent en plus d'une langue naturelle » (1992, p. 15).

16. Selon les conventions du milieu aéronautique

17. Nous avons croisé l'analyse fondée sur la théorie de l'argumentation, du connecteur mais (Ducrot, 1980; Anscombre \& Ducrot, 1983), et en considérant ses homologues pero en espagnol (Ferrer Mora, 2000), aber en allemand (Weydt, 1983) et but en anglais (Lang \& Umbach, 2002), à celle des cadres énonciatifs (Charolles, 1997) qui définissent des univers spatiaux et des univers temporels. Les perspectives des acteurs sont toujours cruciales pour comprendre les langues de communication utilisées au sein d'un groupe, c'est pourquoi les personnes interviewées précisent souvent si elles parlent du contexte de la maison mère, de la multinationale ou de l'organisation, de leur équipe ou de situations avec des clients/partenaires extérieurs.

18. Termes fréquemment entendus dans les formations de formateurs en 2010.

19. Site officiel de la plateforme : Collaboration entre : http://www.speakup-project.eu, consulté le 28/02/13. Les institutions qui ont mis en place le projet sont: Servicio Andaluz de Empleo 
(promoteur du projet) d'Espagne ; Centro Europeu de Linguas du Portugal ; Arbeit und Leben Hamburg d'Allemagne; Foyle International d'Irlande du Nord, Royaume-Uni ; FonsFormacióZonaMediterranea d'Espagne

20. Cette plateforme s'adresse à des apprentis ou des jeunes travailleurs travaillant dans quatre secteurs différents dans l'un des dix métiers suivants: soudeur, installateur de panneaux solaires, serveur / serveuse, employé(e) de maison, réceptionniste d'hôtel, aide-cuisinier, mécanique aéronautique, électronique aéronautique, ouvrier en agriculture biologique, ouvrier viticole.

21. Ce programme a pour vocation de confronter les élèves à la diversité linguistique et d'utiliser cette confrontation pour déclencher des apprentissages. C'est une approche pédagogique qui s'est inspirée des propositions du mouvement Language Awareness développé en GrandeBretagne dans les années 1980 (Hawkins, 1984, 1992 ; Moore, 1995). Deux programmes soutenus par l'Union européenne et le Conseil de l'Europe y ont été consacrés : les programmes Evlang (1997-2000) avec Michel Candelier et Janua Linguarum (2000-2004).

22. La formation communicationnelle a, d'après nous, pour objectif d'englober la dimension linguistique, culturelle et socio-professionnelle. C'est ce vers quoi nous tendons dans cet article.

23. Projet de Réseau Européen de Formateurs (formatrices) à l'IC: Intercompréhension des Langues Romanes http://winterschoolroma-en.weebly.com/prefic.html dernière visite le $07 / 11 / 12$

24. Training Intercomprehension for Cooperation: "The products will be available online, and consist of structured sets of materials organized in different scripts of activities, and a collaborative platform. These scripts, including both face-to-face sessions and online individual tutorials, will be tested in the context of a training course » http:// redinter.wordpress.com/2012/01/31/cinco-training-intercomprehension-for-cooperation/ dernière visite le $07 / 11 / 12$

25. Le projet est financé par le Conseil de l'Europe de novembre 2011 à octobre 2013. Le matériel développé sera mis en ligne fin 2013. Le groupe cible est le personnel maritime. La personne en charge du projet est F. Capucho. http://intermar.ax/?page_id=9 dernière visite le 07/11/12

26. C. Degache décrit les échanges «chats ou clavardages" (1997) comme un premier pas, en 2009 , vers l'oralité puis que ce type de discours est à mi-chemin entre la rigueur de l'écrit et la spontanéité de l'orale. Ces discours sont en fait du « parlécrit » qui n'est ni tout à fait de l'oral, ni tout à fait de l'écrit et pose des problèmes aux apprenants.

\section{RÉSUMÉS}

Parmi les «stratégies de gestion des langues » (PIMLICO Project, 2011) identifiées dans le cadre de la valorisation de compétences linguistiques dans les milieux professionnels internationaux, la formation en langues offre des trajectoires d'apprentissage encore essentiellement monolingue pour des employés pourtant engagés dans une communication quotidienne multilingue, complexe et individuelle.

Cet article part de ce constat pour dresser un état des lieux des formations en langues sur nos deux terrains de recherches qui sont les sites respectifs d'une entreprise aéronautique européenne et de l'UNESCO installés à Hambourg.

Face à un décalage entre la politique linguistique prescrite et les pratiques langagières réelles, nous considérons qu'une approche interdisciplinaire, faisant appel à la linguistique et 
notamment à la question des cadrages ainsi qu'aux sciences de l'éducation et à la sociologie du travail, permet non seulement d'identifier les besoins des salariés, mais aussi d'obtenir du matériel pédagogique issu de corpus d'analyse de discours.

Among the "language management strategies" identified and evaluated in the PIMLICO Project of 2011 in relation to language skills in international professional circles, language training still provides predominantly monolingual learning paths to employees engaged in daily multilingual, complex and individual communication.

This article argues that the development of a language training inventory would be beneficial for both sites of a European aerospace company and UNESCO in Hamburg.

Considering the gap between the prescribed language policy and current language practices, we consider that an interdisciplinary approach, involving a blend of subjects such as linguistics (in particular a "framing" approach), as well as science and the sociology of work would not only satisfy the needs of employees, but would also serve to gather educational materials that could be derived from a corpus of discourse analysis.

\section{INDEX}

Mots-clés : stratégies de gestion des langues, plurilinguisme, milieu professionnel, formation continue

Keywords : language management strategies, multilingualism, workplace, continuous training

\section{AUTEUR}

\section{EVE LEJOT}

Eve Lejot est en poste à l'université du Luxembourg en tant que chargée de cours en linguistique appliquée et en enseignement des langues (français académique). Elle est titulaire d'un doctorat en linguistique de l'université Paris 3 La Sorbonne Nouvelle et de l'université de Hambourg. Elle est membre de l'Institut de recherche sur le multilinguisme et elle est spécialisée dans les analyses de discours et la didactique en langues en contexte multilingue. Entre 2009 et 2012, en parallèle de son activité à l'université de Hambourg, elle a été formatrice en langue dans l'industrie et la diplomatie. Elle a ainsi pu collecter son corpus de recherche.

Courriel : eve.lejot@uni.lu

Adresse : Institut de recherche sur le multilinguisme, Route de Diekirch, 7220 Walferdange, Luxembourg 